\title{
mRNA: a complex(ed) life
}

\author{
Michaela Müller, Karla M Neugebauer* and Christian Eckmann
}

\begin{abstract}
A report of the EMBL conference'The Complex Life of mRNA: From Synthesis to Decay', Heidelberg, Germany, 18-21 March 2010.
\end{abstract}

An EMBL conference held recently in Heidelberg took on the daunting task of examining the life of mRNAs from start to finish. The assumption of the 'central dogma' is that mRNA production leads to protein expression. Yet cellular mRNA levels reflect a balance between the rates of gene transcription and mRNA degradation and translation is highly regulated. Therefore, the level of gene transcription in the cell does not necessarily correlate with encoded protein level. Throughout their lifetimes, all mRNAs exist in complexes with numerous proteins that regulate mRNA at the level of maturation, export, translation and decay. Thus, the life of each mRNA is complex(ed), enabling modulation of gene expression programs beyond promoters. This meeting provided a rare occasion to discuss both mRNA processing and translation regulation in one forum, thereby addressing the central tenets of gene expression in a holistic way. Here we present some of the highlights of the meeting.

\section{The centrality of the poly(A) tail}

During processing in the nucleus, all eukaryotic mRNAs are modified at their $5^{\prime}$ ends by the addition of a 7-methyl guanosine cap and are extended at their 3' ends by addition of a poly(A) tail. These features of mRNA termini are added by enzymatic reactions that are tightly coupled to transcription, ensuring the near universality of these signals, which are crucial for the life of mRNAs in the cytoplasm. Mechanisms of post-transcriptional mRNA regulation exploit the cap and poly(A) tail to control the mRNA's half-life and translational efficiency. Novel cytoplasmic regulatory mechanisms that operate

*Correspondence: neugebau@mpi-cbg.de

Max Planck Institute of Molecular Cell Biology and Genetics, Pfotenhauerstrasse 108, 01307 Dresden, Germany through the poly(A) tail are now coming to light. Numerous microRNAs (miRNAs) repress translation in the context of Argonaute-containing RNA-silencing complexes. The conserved protein GW182 interacts with these RNA-silencing complexes and can trigger translational repression upon tethering to the mRNA. A mechanistic model of how GW182 elicits this response was proposed by Nahum Sonenberg (McGill University, Montreal, Canada). He and colleagues have found that the silencing domain in the human GW182 ortholog TNRC6C interacts with poly(A)-binding protein (PABP), revealing an unexpected role for cytoplasmic PABP as a negative regulator of RNA translation. This model was reinforced and extended by Elisa Izaurralde (Max Planck Institute for Developmental Biology, Tübingen, Germany), who showed that Drosophila GW182 interacts with PABPC1 and may inhibit the closed-loop configuration of the translation initiation factor eIF4G and PABPC1, which stimulate translation synergistically. Importantly, both speakers reported a structural conservation in the GW182/TNRC6C silencing domain that resembles the interaction platform of the well-known PABP inhibitor PAIP2 (PABP-interacting protein 2). Interestingly, although GW182 proteins possess an evolutionarily conserved RNA-silencing function, the molecular details that confer these roles differ. Thus, GW182 is a key player in miRNA-mediated translational repression, which acts by interfering with the stimulatory roles of the $5^{\prime}$ and $3^{\prime}$ ends of mRNA.

The poly(A) tail also has a role in regulating the decay of mRNA. Decay is initiated when the poly(A) tail shrinks below a critical length and the 5' cap is removed by decapping machinery. Deadenylases shorten the poly(A) tail and can directly promote decapping. Bertrand Seraphin (IGBMC, Illkirch, France) revealed the importance of BTG/TOB proteins as a novel class of conserved mRNA decay regulators in metazoans. BTG/TOB proteins bind directly to the CAF1 subunit of the CCR4/CAF1/ Not complex, an assembly that contains multiple deadenylases. BTG/TOB proteins regulate the general deadenylation of mRNAs and thereby contribute to several post-transcriptional control mechanisms, including development and the control of cell proliferation. A second example of such post-transcriptional regulation was provided through Seraphin's analysis of the role of 
yeast AU-rich element binding proteins, which were, surprisingly, shown to control alternative polyadenylation in addition to mRNA decay.

All classes of RNA are subject to mechanisms of surveillance, which remove aberrant or nonfunctional RNAs. David Tollervey (Wellcome Trust Centre for Cell Biology, University of Edinburgh, UK) presented work on the yeast Nrd1/Nab3 surveillance complex, which may recognize cryptic noncoding RNAs and cryptic unstable transcripts via a short poly $(\mathrm{A})$ tail of four adenosines and target them for rapid decay mediated by the TRAMP complex. Tollervey introduced cross-linking and analysis of cDNAs (CRAC) as a tool for identifying ribonucleoproteins (RNPs) at the whole-transcriptome level. He reported that long antisense noncoding RNAs and nascent rRNA transcripts of stalled RNA polymerase I complexes are also targets of the Nrd1/Nab3-TRAMP surveillance complex. With the advance of RNA sequencing technologies, we have only begun to understand the world of nonproductive RNA synthesis and the duties of RNA surveillance machineries.

Poly(A) tail elongation by noncanonical poly(A) polymerase complexes is known to stimulate RNA translation. Raúl Méndez (CRG, Barcelona, Spain) presented exciting work on the expanding biological roles of the cytoplasmic polyadenylation element binding proteins (CPEBs). CPEBs are important for cytoplasmic polyadenylation, regulate translation, and have now been found to also control the progression of the mitotic cell cycle. Mendez described how cell-cycle-specific changes in poly(A) tail length are important for cell division. Deregulation of this process may occur in tumors and result in abnormal expression of factors involved in tumor progression. Originally, regulation of poly(A) tail length was shown to be heavily used by female germ cells undergoing programmed developmental arrest in their normal meiotic program. This new work shows the versatility of cytoplasmic polyadenylation as an important regulatory mechanism in health and disease.

\section{Binding sites for small RNAs: the importance of location}

In general, miRNAs interact with binding sites localized in the 3' UTR of target mRNAs. Matthias Hentze (EMBL, Heidelberg, Germany) reported that the mechanism of translational repression by Drosophila miR-2 is principally independent of the position of miRNA-binding sites. When a reporter mRNA with six neighboring miR2-binding sites was used, miRNA targeting to the 5' UTR, coding sequence or 3' UTR was equally effective at repressing translation. Interestingly, when the miR-2binding sites were progressively reduced to one or two, reflecting the most common physiological settings, translational repression became sensitive to position;
miRNA-mediated regulation was only observed when the single binding site was located in the 3' UTR. In contrast, the liver-specific miR-122 binds to two adjacent sites close to the 5 ' end of the hepatitis $\mathrm{C}$ virus (HCV) RNA genome; miR-122 binding results in upregulation of viral RNA levels and propagation of the virus. Peter Sarnow (Stanford University, Stanford, USA) reported that insertion of the viral miR-122 binding site into the 3' noncoding region of a reporter mRNA led to a decrease in mRNA expression in human tissue culture cells. Both examples illustrate how the location of miRNA binding sites dictates their effects on gene regulation.

In contrast to the eukaryotic miRNAs, the binding sites of small bacterial RNAs (sRNAs) that repress translation are mainly localized in the 5' UTR of the mRNA in close proximity to the Shine-Dalgarno (SD) sequence. This is consistent with the known role of sRNAs in regulation of loading of mRNAs onto ribosomes. However, Jörg Vogel (Max Planck Institute for Infection Biology, Berlin, Germany) described how sRNA-binding sites are common outside the canonical SD region, even far upstream in the 5' UTR or within the coding sequence. He revealed that target sites in the coding sequence suggest that sRNAs can silence genes downstream of translational initiation, probably by promoting mRNA degradation.

\section{Regulating the regulators}

Diverse Argonaute (Ago) proteins bind various classes of small RNAs (for example, miRNAs, small interfering RNAs (siRNAs), and PIWI-interacting RNAs (piRNAs)) to form RNA-silencing complexes and act as binding platforms for post-transcriptional mRNA regulatory complexes. Surprisingly little is known about the regulation of Ago proteins themselves and the complexes they form. Gunter Meister (University of Regensburg, Regensburg, Germany) presented work on human Ago2 protein, which is phosphorylated on a conserved tyrosine; phosphorylation reduces the RNA-binding potential of Ago2 but leaves all other aspects of Ago2 functions intact. In contrast, germline-enriched PIWI proteins are known to contain symmetrically dimethylated arginine. Mikiko Siomi (Keio University, Tokyo, Japan) has discovered that arginine methylation enhances Ago protein complex formation and the efficient loading of small RNAs in Drosophila. Interestingly, Siomi reported that tudor-domain proteins are sensitive to argininemodified Ago proteins and may therefore represent key regulators of piRNA-directed silencing complexes. Thus, signaling cascades may regulate post-transcriptional controls during development or in response to changing environmental conditions.

Although miRNAs are involved in regulatory processes, paradoxically they have long half-lives, sometimes longer 
than 12 hours. Two talks featured the importance of rapid turnover for miRNAs in specific biological contexts. Witold Filipowicz (Friedrich Miescher Institute for Biomedical Research, Basel, Switzerland) reported that many miRNAs in retinal and non-retinal neurons have very short half-lives and that miRNA turnover is regulated by neuronal activity. He also described identification of miRNAs in mouse retina, the abundance of which is controlled by exposure to light. Sebastien Pfeffer (IBMC, Strasbourg, France) reminded us that the abundance of specific cellular miRNAs changes upon infection with viruses, which can have an impact on viral production and infectivity. Pfeffer reported the rapid downregulation of miR-27, a cellular miRNA that targets murine cytomegalovirus (MCMV) and exerts an antiviral function when overexpressed. Pfeffer has found that downregulation of miR-27 occurs at the posttranscriptional level by the induction of rapid decay of the mature miRNA.

\section{Open and closed conformations facilitate function}

Structure-guided functional studies provide mechanistic insights into all aspects of RNA biology. Conformational changes in the core machineries emerged as a common theme. Patrick Cramer (Gene Center, University of Munich, Germany) presented the structure of yeast RNA polymerase II bound to the transcription initiation factor TFIIB. He discussed models for the closed and open promoter complexes in the context of transcription initiation and the transition to elongation. Cramer showed that the 'B-linker' region of TFIIB is essential for DNA opening, while the 'B-reader' region is important for transcription start-site selection. Ribosome function is also dependent on open and closed conformations. Alan Hinnebusch (National Institute of Child Health and Human Development, NIH, Bethesda, USA) reported the identification of multiple motifs (SI1, SI2, SE1 and SE2) in the amino- and carboxy-terminal tails of the translation initiation factor eIF1A. He showed that the SE elements enhance binding of the ternary complex to the $40 \mathrm{~S}$ ribosome in a conformation conducive to scanning but incompatible with start codon selection (open scanning conformation). SI elements antagonize SE elements to destabilize this open conformation of the ribosome and promote the transition to a closed, scanning-incompatible conformation with tRNA ${ }^{\mathrm{i} \text { et }}$ fully engaged in the P-site for AUG selection.

Riboswitches are natural RNA sensors that respond to intracellular metabolites and ions in order to control gene expression by controlling translation efficiency, transcription termination or RNA stability. Dinshaw Patel (Memorial Sloan-Kettering Cancer Center, New York, USA) presented the structure of the glycine riboswitch with ligand (closed conformation) and without ligand (open conformation). Patel explained that in contrast to all other known amino-acid riboswitches, the glycine riboswitch contains only one sensing domain and in this way discriminates against larger amino acids.

The exon-junction complex (EJC) is a key player in post-transcriptional gene regulation in metazoans. This multiprotein complex is loaded onto mRNAs upon splicing and modulates nuclear mRNA export, nonsensemediated decay (NMD) and translation efficiency of mRNAs. Elena Conti (Max Planck Institute for Biochemistry, Munich, Germany) presented the crystal structure of the mammalian core EJC complex bound to the NMD effector protein UPF3. She showed how UPF3 bridges the UPF and EJC complexes by interacting with the EJC components Mago, Y14 and eIF4AIII (closed conformation). Conti showed that eIF4AIII and Y14 have hotspots for interactions on their surface that are used to recruit different regulators. The shuttling Mago-Y14 heterodimer binds the EJC disassembly factor PYM in the cytoplasm. PYM binds to the same pocket in the Mago-Y14 heterodimer as UPF3, suggesting a mechanism whereby mutually exclusive binding sites impart a sequential order in the interactions in the NMD pathway. Niels Gehring (EMBL, Heidelberg, Germany) has investigated whether the deposition of the EJC influences the association of the mammalian mRNA export complex TREX with mRNAs. Although TREX recruitment to the 5' end of the mRNA was independent of splicing, he could demonstrate that the EJC significantly contributes to the recruitment of the TREX complex to downstream sites, which could serve as a failsafe mechanism for TREX recruitment.

Two key themes emerged from the meeting: first, the coding potential of genes is extensively regulated posttranscriptionally at numerous stages in an mRNA's life; second, the protein complexes that meet mRNA couple each life stage sequentially with one another, such that transcription influences processing, processing influences export, export influences stability and translation, and so on. Several examples now illustrate how RNA-protein complexes undergo switching behavior to determine an mRNA's fate, depending on the biological context. This complexity is a clear endorsement for future meetings like this one, addressing gene expression from the point of view of the mRNA's lifetime.

Published: 25 May 2010

doi:10.1186/gb-2010-11-5-304

Cite this article as: Müller M, et al:: mRNA: a complex(ed) life. Genome Biology 2010, 11:304 